\title{
MAGNETIC FIELD OF LATE-TYPE STARS: A NEW APPROACH *
}

\author{
G. Mathys
}

Geneva Observatory, ch. des Maillettes 51, CH-1290 Sauverny, Switzerland

S.K. Solanki

Institute of Astronomy, ETH-Zentrum, $\mathrm{CH}-8092$ Zurich, Switzerland

ABSTRACT. Magnetic field diagnosis in three late-type stars is performed using the multiline technique originally developed by Stenflo and Lindegren (1977) to study spatially unresolved magnetic features on the sun.

We present in this paper a preliminary report of the application of a new method to the diagnosis of magnetic fields in late-type stars: the multiline technique first developed by Stenflo and Lindegren (1977) for the study of the spatially unresolved structure of solar magnetic fields. In this approach, the profiles of a large number of simultaneously recorded spectral lines are characterized by some simple parameters whose mutual correlations and dependences on atomic parameters such as the excitation potential of the lower level of the transition or the effective Lande factor are empirically determined through a regression analysis.

The observations have been performed with the ESO Coude Echelle Spectrometer fed by the Coudé Auxiliary Telescope. The long camera was used with the RETICON to achieve a spectral resolution of 100000 . Each star was consecutively observed in several wavelength ranges over the same night, so that a sufficient number of lines are available to apply the statistical analysis sketched above. For each star, we study the largest possible sample of apparently unblended Fe I lines. Details about the observations are given in Table 1 . Columns 1 and 2 give the star's name and HR number, and Col. 3 the $V$ magnitude. The duration of observation listed in Col. 5 refers to the interval of time elapsed between the beginning of the exposure of the first wavelength range and the end of the exposure of the last wavelength range, and the date of the observation in Col. 4 corresponds to the middle of that time interval. In Col. 6 we give an estimate of the achieved $\mathrm{S} / \mathrm{N}$, for the wavelength domains with the lowest and the highest quality recording.

Table 1. Observations of late-type stars with the CES at the CAT

\begin{tabular}{|c|c|c|c|c|c|c|}
\hline Name & HR & $V$ & Sp. & $\begin{array}{c}\text { Date } \\
\text { HJD 2 440 000.+ }\end{array}$ & $\begin{array}{c}\text { Duration } \\
\text { (hours) }\end{array}$ & $S / N$ \\
\hline$\tau$ Cet & 509 & 3.50 & G8V & 6718.70 & 8.4 & $500-700$ \\
$\epsilon$ Eri & 1084 & 3.73 & K2V & 6722.76 & 4.7 & $250-400$ \\
40 Eri A & 1325 & 4.42 & K1V & 6723.75 & 5.8 & $300-400$ \\
\hline
\end{tabular}

The parameters characterizing the line profiles are (Stenflo and Lindegren, 1977): (i) the line depth $d$, (ii) the chord length $v_{D}(z)$ at level $z d(0<z<1)$ above the line bottom (expressed in

* Based on observations collected at the European Southern Observatory, La Silla, Chile 
velocity units in terms of the formal Doppler width of a Gaussian profile that has the same width as the considered line at the given chord level), and (iii) the area $S$ of the line below the half level chord (a line strength parameter, less sensitive to blends than the equivalent width).

The following regression equation proves to be suitable for the line width at any level in the

line:

$$
v_{D}(z)=x_{0}+x_{1} v_{m}^{2} \lambda^{2} / v_{0}+x_{2}\left\langle v_{m}^{2}\right\rangle \lambda^{2} / v_{0}+x_{3} S+x_{4} S^{2}+x_{5} \chi_{e} v_{0},
$$

where $x_{0}, \ldots, x_{5}$ are the regression coefficients, $\lambda$ is the line wavelength, $\chi_{e}$ is the excitation potential of the lower level of the transition, $v_{0}=y_{0}+y_{1} S^{2}$ is an approximation of $v_{D}$ (see Stenflo and Lindegren, 1977, for more details), and

$$
v_{m}^{2}=\left(\bar{g}^{2}+X_{\sigma}\right) \frac{1+\cos ^{2} \gamma}{2}+X_{\pi} \frac{\sin ^{2} \gamma}{2}
$$

for a magnetic field at an angle $\gamma$ to the line of sight (Mathys and Stenflo, 1987a). $\bar{g}$ is the effective Lande factor of the transition, and $X_{\pi}$ and $X_{\sigma}$ are the second order moments about their centre of gravity of the $\pi$ and the $\sigma_{+}$components of the Zeeman pattern, respectively, expressed in units of the Zeeman splitting of a normal triplet having a Lande factor equal to 1 (Landi Degl'Innocenti, 1982, 1985; Mathys and Stenflo, $1987 \mathrm{a}, \mathrm{b})$. The regression coefficient $x_{1}$ is related to the magnetic field strength $B$ through:

$$
x_{1}=k^{2} c^{2} \alpha \delta_{c} \delta_{l} B^{2},
$$

where $k=4.6710^{-13} \AA^{-1} \mathrm{G}^{-1}, c$ is the velocity of light, $\alpha$ is the filling factor, $\delta_{c}$ is the continuum contrast of the magnetic regions relative to the nonmagnetic regions, and $\delta_{l}$ is the average ratio of line strength in magnetic regions to that in nonmagnetic regions.

The regression equation for the line depth $d$ is similar to Eq. (1). However tests have shown that the line width regression is a better diagnostic for magnetic field properties. One reason for this is that the noise is greater in the line cores, which are formed at different levels in the atmosphere, than in the line flanks, which are all formed at nearly the same height.

From Eq. (3), one sees that the relation between $x_{1}$ and $B$ involves three other a priori unknown quantities, $\alpha, \delta_{c}$ and $\delta_{l}$. Let us now discuss to which extent these quantities and the field strength can be disentangled using the Stenflo-Lindegren technique. For a filling factor less than 1 , the part of the line nearer the continuum is more strongly affected by the magnetic field than the line core, thus e.g. the ratio $x_{1}(z=0.7) / x_{1}(z=0.5)$ is a measure of the product $\alpha \delta_{c} \delta_{l}$ as has been illustrated in the case of the sun by Brandt and Solanki (1987). On the other hand, the multiline approach also provides the possibility of deciding whether the magnetic fields are concentrated in hot or in cool regions of the stellar surface, i.e. in stellar plages or spots. Indeed, in the former case, the low excitation lines are weakened with respect to the high excitation lines in the magnetic regions compared with the nonmagnetic regions. The opposite will be the case if the magnetic fields are concentrated in cool elements. Thus, sorting the lines into a high excitation and a low excitation group, $\delta_{l}$ will be different for these two groups. Since the product $\alpha \delta_{c} B^{2}$ is approximately the same for both groups, one has:

$$
\frac{x_{1}^{\text {high }}}{x_{1}^{\text {low }}}=\frac{\delta_{1}^{\text {high }}}{\delta_{1}^{\text {low }}} .
$$

Hence performing the regression for both groups of lines separately, the difference in the obtained coefficient $x_{1}$ is ideally a measure of the difference in temperature between the magnetic elements and their nonmagnetic surroundings (provided that the $\mathrm{S} / \mathrm{N}$ and statistics are good enough). On the other hand, it has recently been shown in the case of the sun (Grossmann-Doerth et al., 1987; Schüssler and Solanki, 1987) that in the absence of resolved magnetic features, no method exists (either polarimetric or not) that permits the determination of $\alpha$ directly: only $\alpha \delta_{c}$ is defined.

The results that we obtain are summarized in table 2 . Column 3 gives the number of $\mathrm{Fe}$ I lines used in the analysis. The quantities listed in Cols. 4 to 7 were derived assuming a mean 
Table 2. Derived magnetic field values

\begin{tabular}{|c|c|c|c|c|c|c|}
\hline Name & HR & $\begin{array}{c}\text { No.of } \\
\text { lines }\end{array}$ & $\begin{array}{c}\sqrt{\alpha \delta_{c} \delta_{l}} B \\
(\mathrm{G})\end{array}$ & $\frac{\sqrt{\alpha \delta_{c} \delta_{l}} B}{\sigma\left(\sqrt{\alpha \delta_{c} \delta_{l}} B\right)}$ & $\alpha \delta_{c} \delta_{l}$ & $\begin{array}{c}B \\
(\mathrm{G})\end{array}$ \\
\hline$\tau$ Cet & 509 & 65 & 220 & 1.2 & & \\
$\epsilon$ Eri & 1084 & 45 & 800 & 4.6 & $0.10-0.20$ & $\begin{array}{c}1790-2530 \\
50 \text { Eri A }\end{array}$ \\
\hline
\end{tabular}

angle between the line of sight and the magnetic field $\gamma=34^{\circ}$ in Eq. (2). Note that in principle the magnetic term of the regression equation (1) could be split into two and $\gamma$ could be diagnosed independently, taking advantage of the presence of various Zeeman patterns in the line sample. In HR 509, there is only marginal evidence of detection of a magnetic field. For the other two stars, HR 1084 and HR 1325, for which the presence of a magnetic field is more certain, we also set some constraints on the value of the product $\alpha \delta_{c} \delta_{l}$ and on the related value of $B$. Finally, in HR 1084, sorting the lines into two categories according to the excitation potential $\left(\chi_{e}^{\text {low }} \leq 3 \mathrm{eV}, \chi_{e}^{\text {high }} \geq 4 \mathrm{eV}\right)$, we obtain $\delta_{l}^{\text {high }} / \delta_{l}^{\text {low }} \approx 2.0$, indicating that the observed magnetic field is concentrated in regions that are hotter than the nonmagnetic part of the atmosphere. These various results are compatible with those obtained previously by other techniques (Marcy, 1984; Gray, 1984; Saar, 1987). This is discussed in more detail by Solanki and Mathys (1987).

To summarize, we have presented here a preliminary account of the application of the StenfloLindegren technique to the diagnosis of the magnetic field of late-type stars. The advantages of this method over the previously used ones are its relative unsensitivity to line blends and its ability to set constraints on the temperature difference between magnetic and nonmagnetic regions of the atmosphere. For the future, the following developments of the present work are envisaged:

(i) To compare the results that we obtain with those of other methods, applied to the same data.

(ii) To investigate possible systematic effects of the line blends on the results.

(iii) To study the influence of the time spread of the observations (note in this respect that since the various wavelength ranges were not recorded in order of increasing or decreasing wavelength or effective Landé factor, rapid changes in $\sqrt{\alpha \delta_{c} \delta_{l}} B$ essentially increase the scatter about the regression).

(iv) To try to set constraints on the magnetic geometry.

(v) To observe other stars, in particular in the southern hemisphere where no systematic survey has up to now be performed.

\section{References}

Brandt, P.N., Solanki, S.K.: 1987, Astron. Astrophys. (to be submitted)

Gray, D.F.: 1984, Astrophys. J. 277, 640

Grossmann-Doerth, U., Pahlke, K.-D., Schüssler, M.: 1987, Astron. Astrophys. 176, 139

Landi Degl'Innocenti, E.: 1982, Solar Phys. 77, 285

Landi Degl'Innocenti, E.: 1985, Solar Phys. 99, 1

Marcy, G.W.: 1984, Astrophys. J. 276, 286

Mathys, G., Stenflo, J.O.: 1987a, Astron. Astrophys. 171, 368

Mathys, G., Stenflo, J.O.: 1987b, Astron. Astrophys. Suppl. 67, 557

Saar, S.H.: 1987, Ph. D. Thesis, JILA, Boulder

Schüssler, M., Solanki, S.K.: 1987, Astron. Astrophys. (in press)

Solanki, S.K., Mathys, G.: 1987, in Proceedings of the Midnight Sun Conference on Activity in Cool Star Envelopes, Troms $\varnothing$, Norway, in press 
DIscussion

NISSEN How faint magnitudes can you reach with CASPEC using
this technique?
mirys You can in principle go as faint with the zeeman
analyzer as in the standard mode of the CASPEC, expect for a factor of
about two (as a result of the splitting of the beam into two
polarizations). That is, for a s/N of 100 or higher, it should be
possible to reach magnitude 13 . Our main limitation comes rather from the
mild resolving power of the CASPEC. 